Supplement of Hydrol. Earth Syst. Sci., 21, 3221-3229, 2017

https://doi.org/10.5194/hess-21-3221-2017-supplement

(C) Author(s) 2017. This work is distributed under

the Creative Commons Attribution 3.0 License.

(c) (1)

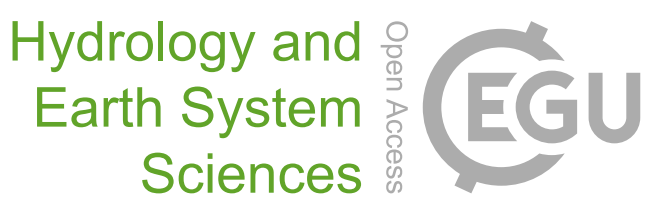

Supplement of

\title{
Slope-velocity equilibrium and evolution of surface roughness on a stony hillslope
}

Mark A. Nearing et al.

Correspondence to: Mark A. Nearing (mark.nearing@ars.usda.gov)

The copyright of individual parts of the supplement might differ from the CC BY 3.0 License. 


\section{Supplement}

1: Runoff Ratios a function of cumulative runoff.

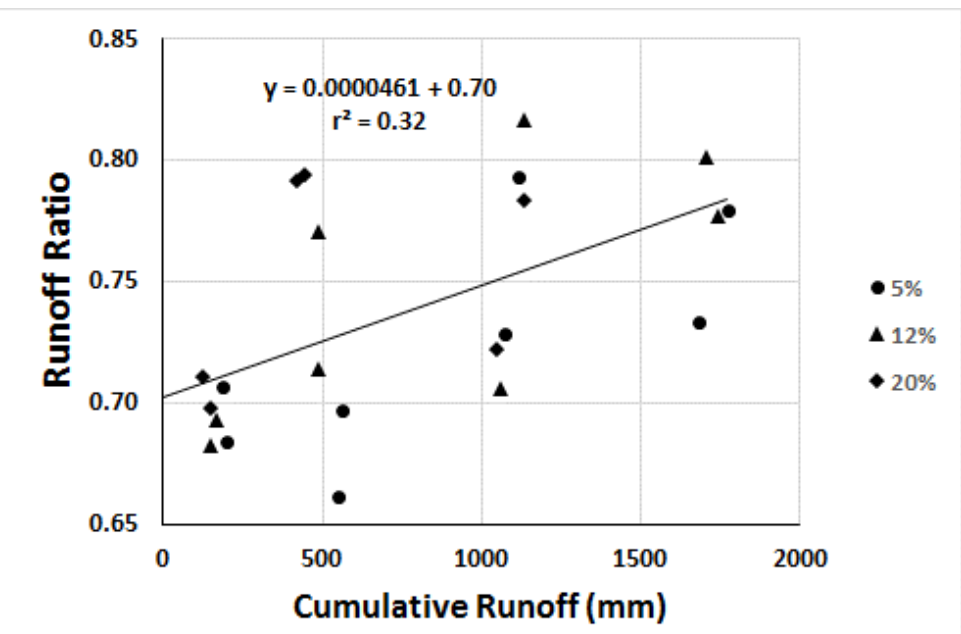

2: Schematic of an experimental sequence for Replication 1 of the $12 \%$ slope.

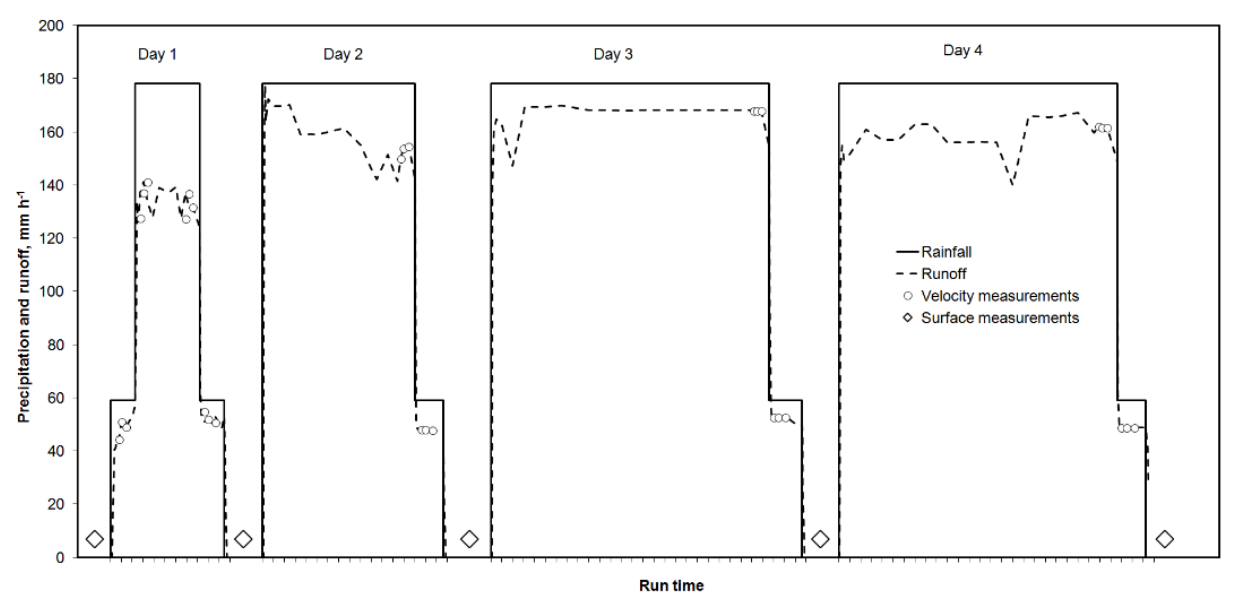

3: Photo of the experiment in progress. Taken during run 3 at 20\% slope, replication \#1. 


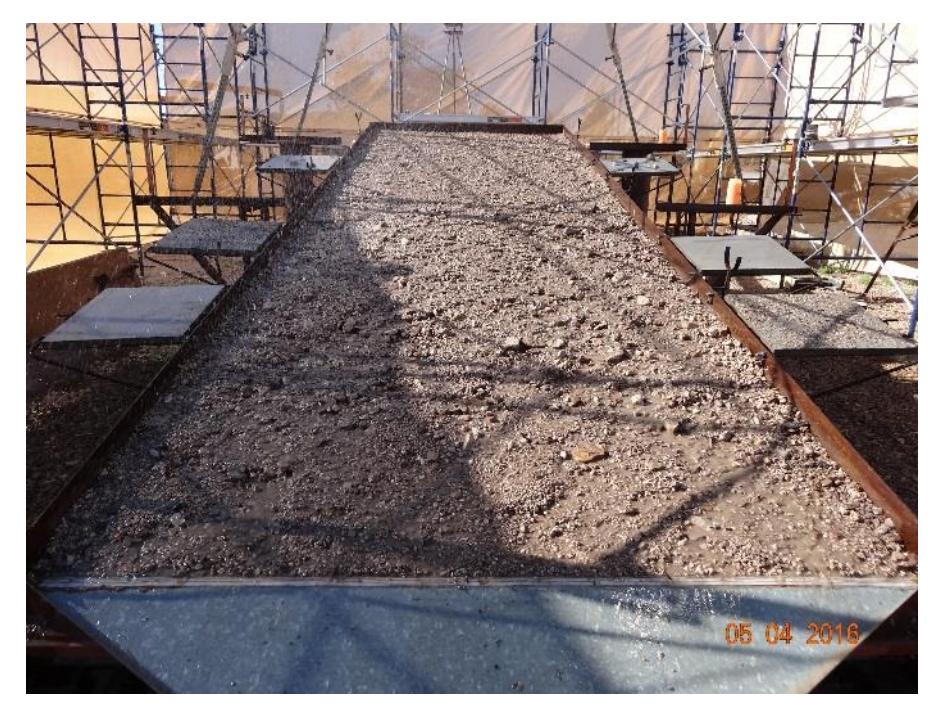

4: Example of photo of rock cover. Taken after run 3 at 20\% slope, replication \#2.

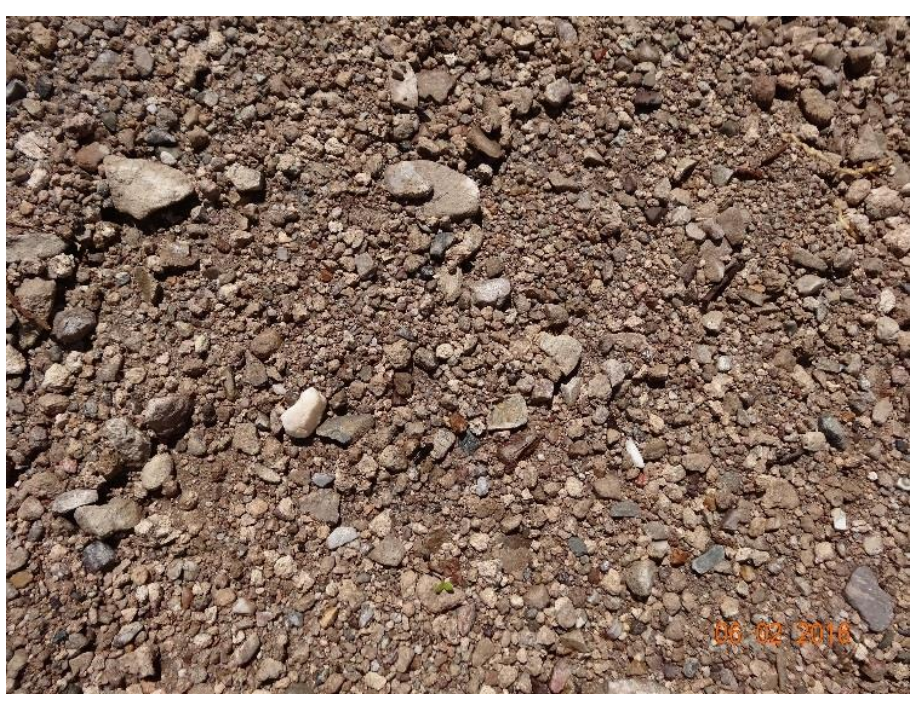




\section{5: Data}

Slope Rep Run Rainfall Distance Velocity

Section Section Chezy Manning's Meas. Meas Interp. Interp Peak Rock

\begin{tabular}{|c|c|c|c|c|c|c|c|c|c|c|c|c|c|c|c|}
\hline$\%$ & $\#$ & $\#$ & $\begin{array}{l}\mathrm{Int} . \\
\mathrm{mm} / \mathrm{h}\end{array}$ & $\mathrm{m}$ & $\begin{array}{l}\text { Peak } \\
\mathrm{m} / \mathrm{s}\end{array}$ & $\begin{array}{l}\text { Runoff } \\
\mathrm{l} / \mathrm{min}\end{array}$ & $\begin{array}{l}\text { Runoff } \\
\mathrm{mm}\end{array}$ & $\begin{array}{l}\text { Vel. } \\
\mathrm{m} / \mathrm{s}\end{array}$ & $\begin{array}{l}\text { Discharge } \\
\mathrm{l} / \mathrm{min}\end{array}$ & $\begin{array}{l}m^{\wedge} 1 / 2 \\
s^{\wedge}-1 \\
\end{array}$ & $s \mathrm{~m}^{\wedge}-1 / 3$ & $\begin{array}{l}\text { Rough. } \\
\mathrm{mm}\end{array}$ & $\begin{array}{l}\mathrm{C} \\
\% \\
\end{array}$ & $\begin{array}{l}\text { Rough } \\
\mathrm{mm}\end{array}$ & $\begin{array}{l}\text { Rock } \\
\% \\
\end{array}$ \\
\hline 5 & 1 & 0 & 59 & 1.65 & 0.046 & 8.35 & 2.6 & 0.0465 & 7.22 & 5.779 & 0.057 & 2.09 & 40 & 2.06 & 43 \\
\hline 5 & 1 & 0 & 59 & 3.5 & 0.036 & 8.56 & 5.8 & 0.0305 & 4.94 & 3.706 & 0.090 & 2.03 & 23 & 2.19 & 28 \\
\hline 5 & 1 & 0 & 59 & 5.8 & 0.026 & 8.72 & 9.3 & 0.0177 & 2.05 & 2.552 & 0.123 & 2.33 & 32 & 2.38 & 35 \\
\hline 5 & 1 & 0 & 178 & 1.65 & 0.090 & 25.72 & 24.4 & 0.0898 & 22.23 & 8.845 & 0.040 & 2.09 & 40 & 2.00 & 47 \\
\hline 5 & 1 & 0 & 178 & 3.5 & 0.072 & 25.72 & 29.7 & 0.0609 & 14.83 & 6.046 & 0.059 & 2.03 & 23 & 2.38 & 33 \\
\hline 5 & 1 & 0 & 178 & 5.8 & 0.047 & 25.72 & 37.1 & 0.0305 & 6.05 & 3.357 & 0.102 & 2.33 & 32 & 2.41 & 38 \\
\hline 5 & 1 & 1 & 178 & 1.65 & 0.080 & 26.37 & 150.9 & 0.0800 & 22.79 & 7.345 & 0.050 & 1.77 & 65 & 1.86 & 58 \\
\hline 5 & 1 & 1 & 178 & 3.5 & 0.068 & 26.37 & 155.2 & 0.0594 & 15.20 & 5.756 & 0.062 & 3.23 & 56 & 2.92 & 48 \\
\hline 5 & 1 & 1 & 178 & 5.8 & 0.046 & 27.34 & 164.1 & 0.0314 & 6.43 & 3.398 & 0.102 & 2.59 & 53 & 2.53 & 48 \\
\hline 5 & 1 & 1 & 59 & 1.65 & 0.048 & 9.34 & 181.8 & 0.0480 & 8.08 & 5.734 & 0.058 & 1.77 & 65 & 1.82 & 61 \\
\hline 5 & 1 & 1 & 59 & 3.5 & 0.039 & 9.34 & 185.0 & 0.0340 & 5.39 & 4.188 & 0.079 & 3.23 & 56 & 3.07 & 52 \\
\hline 5 & 1 & 1 & 59 & 5.8 & 0.024 & 8.64 & 189.3 & 0.0155 & 2.03 & 2.102 & 0.153 & 2.59 & 53 & 2.57 & 51 \\
\hline 5 & 1 & 2 & 178 & 1.65 & 0.071 & 25.15 & 511.6 & 0.0710 & 21.73 & 6.283 & 0.059 & 2.68 & 74 & 2.49 & 72 \\
\hline 5 & 1 & 2 & 178 & 3.5 & 0.062 & 25.15 & 516.8 & 0.0561 & 14.50 & 5.401 & 0.067 & 2.47 & 76 & 2.62 & 72 \\
\hline 5 & 1 & 2 & 178 & 5.8 & 0.039 & 25.15 & 524.0 & 0.0245 & 5.92 & 2.447 & 0.145 & 2.72 & 83 & 2.70 & 77 \\
\hline 5 & 1 & 2 & 59 & 1.65 & 0.046 & 9.51 & 541.0 & 0.0465 & 8.22 & 5.415 & 0.062 & 2.68 & 74 & 2.57 & 73 \\
\hline 5 & 1 & 2 & 59 & 3.5 & 0.034 & 9.51 & 544.5 & 0.0280 & 5.48 & 3.105 & 0.110 & 2.47 & 76 & 2.55 & 74 \\
\hline 5 & 1 & 2 & 59 & 5.8 & 0.023 & 9.21 & 549.5 & 0.0156 & 2.17 & 2.044 & 0.159 & 2.72 & 83 & 2.71 & 81 \\
\hline 5 & 1 & 3 & 178 & 1.65 & 0.069 & 26.29 & 1016.7 & 0.0688 & 22.73 & 5.858 & 0.064 & 2.87 & 74 & 2.84 & 74 \\
\hline 5 & 1 & 3 & 178 & 3.5 & 0.058 & 26.29 & 1023.1 & 0.0514 & 15.16 & 4.635 & 0.079 & 2.81 & 75 & 2.76 & 75 \\
\hline 5 & 1 & 3 & 178 & 5.8 & 0.037 & 26.29 & 1032.9 & 0.0242 & 6.19 & 2.351 & 0.153 & 2.79 & 82 & 2.78 & 82 \\
\hline 5 & 1 & 3 & 59 & 1.65 & 0.045 & 9.92 & 1052.4 & 0.0446 & 8.58 & 4.981 & 0.069 & 2.87 & 74 & 2.86 & 74 \\
\hline 5 & 1 & 3 & 59 & 3.5 & 0.035 & 9.92 & 1056.1 & 0.0289 & 5.72 & 3.174 & 0.108 & 2.81 & 75 & 2.79 & 75 \\
\hline 5 & 1 & 3 & 59 & 5.8 & 0.022 & 9.87 & 1060.9 & 0.0144 & 2.32 & 1.762 & 0.188 & 2.79 & 82 & 2.79 & 82 \\
\hline 5 & 1 & 4 & 178 & 1.65 & 0.061 & 26.91 & 1627.5 & 0.0611 & 23.26 & 4.853 & 0.079 & 3.22 & 90 & 3.17 & 88 \\
\hline 5 & 1 & 4 & 178 & 3.5 & 0.058 & 26.91 & 1634.2 & 0.0548 & 15.52 & 5.047 & 0.072 & 2.89 & 92 & 2.88 & 90 \\
\hline 5 & 1 & 4 & 178 & 5.8 & 0.038 & 26.84 & 1643.0 & 0.0247 & 6.31 & 2.398 & 0.150 & 2.60 & 88 & 2.63 & 87 \\
\hline 5 & 1 & 4 & 59 & 1.65 & 0.037 & 9.92 & 1661.0 & 0.0368 & 8.58 & 3.729 & 0.095 & 3.22 & 90 & 3.19 & 89 \\
\hline 5 & 1 & 4 & 59 & 3.5 & 0.029 & 9.95 & 1665.1 & 0.0247 & 5.74 & 2.506 & 0.141 & 2.89 & 92 & 2.89 & 91 \\
\hline 5 & 1 & 4 & 59 & 5.8 & 0.021 & 9.95 & 1670.8 & 0.0145 & 2.34 & 1.761 & 0.189 & 2.60 & 88 & 2.61 & 88 \\
\hline 5 & 2 & 0 & 59 & 1.65 & 0.049 & 7.29 & 7.3 & 0.0491 & 6.30 & 6.707 & 0.048 & 2.65 & 23 & 2.59 & 28 \\
\hline 5 & 2 & 0 & 59 & 3.5 & 0.045 & 7.29 & 9.4 & 0.0425 & 4.20 & 6.630 & 0.046 & 2.42 & 20 & 2.41 & 26 \\
\hline 5 & 2 & 0 & 59 & 5.8 & 0.027 & 7.29 & 12.7 & 0.0168 & 1.72 & 2.566 & 0.120 & 1.94 & 38 & 2.05 & 40 \\
\hline
\end{tabular}




\begin{tabular}{|c|c|c|c|c|c|c|c|c|c|c|c|c|c|c|c|}
\hline 5 & 2 & 0 & 178 & 1.65 & 0.094 & 26.56 & 28.7 & 0.0936 & 22.96 & 9.257 & 0.038 & 2.65 & 23 & 2.51 & 35 \\
\hline 5 & 2 & 0 & 178 & 3.5 & 0.083 & 27.58 & 34.7 & 0.0748 & 15.90 & 7.945 & 0.044 & 2.42 & 20 & 2.40 & 32 \\
\hline 5 & 2 & 0 & 178 & 5.8 & 0.050 & 27.58 & 43.0 & 0.0313 & 6.49 & 3.367 & 0.103 & 1.94 & 38 & 2.13 & 41 \\
\hline 5 & 2 & 1 & 178 & 1.65 & 0.073 & 24.88 & 139.7 & 0.0733 & 21.50 & 6.634 & 0.055 & 2.24 & 63 & 2.36 & 51 \\
\hline 5 & 2 & 1 & 178 & 3.5 & 0.069 & 26.76 & 145.0 & 0.0649 & 15.43 & 6.523 & 0.054 & 2.36 & 54 & 2.37 & 44 \\
\hline 5 & 2 & 1 & 178 & 5.8 & 0.044 & 26.76 & 153.8 & 0.0281 & 6.30 & 2.914 & 0.120 & 2.45 & 45 & 2.32 & 43 \\
\hline 5 & 2 & 1 & 59 & 1.65 & 0.038 & 8.53 & 172.1 & 0.0384 & 7.38 & 4.288 & 0.080 & 2.24 & 63 & 2.31 & 56 \\
\hline 5 & 2 & 1 & 59 & 3.5 & 0.038 & 8.53 & 175.3 & 0.0379 & 4.92 & 5.163 & 0.062 & 2.36 & 54 & 2.37 & 49 \\
\hline 5 & 2 & 1 & 59 & 5.8 & 0.024 & 8.53 & 179.8 & 0.0154 & 2.01 & 2.091 & 0.153 & 2.45 & 45 & 2.40 & 44 \\
\hline 5 & 2 & 2 & 178 & 1.65 & 0.063 & 23.27 & 486.1 & 0.0629 & 20.11 & 5.444 & 0.068 & 2.51 & 70 & 2.44 & 68 \\
\hline 5 & 2 & 2 & 178 & 3.5 & 0.057 & 23.27 & 492.7 & 0.0530 & 13.41 & 5.168 & 0.069 & 2.50 & 74 & 2.47 & 69 \\
\hline 5 & 2 & 2 & 178 & 5.8 & 0.041 & 23.45 & 504.3 & 0.0287 & 5.52 & 3.200 & 0.107 & 2.62 & 65 & 2.58 & 61 \\
\hline 5 & 2 & 2 & 59 & 1.65 & 0.038 & 9.56 & 525.1 & 0.0384 & 8.27 & 4.050 & 0.086 & 2.51 & 70 & 2.47 & 69 \\
\hline 5 & 2 & 2 & 59 & 3.5 & 0.035 & 9.56 & 529.1 & 0.0318 & 5.51 & 3.746 & 0.090 & 2.50 & 74 & 2.48 & 72 \\
\hline 5 & 2 & 2 & 59 & 5.8 & 0.023 & 9.56 & 535.0 & 0.0148 & 2.25 & 1.862 & 0.177 & 2.62 & 65 & 2.60 & 63 \\
\hline 5 & 2 & 3 & 178 & 1.65 & 0.070 & 25.95 & 1048.8 & 0.0698 & 22.43 & 6.038 & 0.062 & 2.65 & 78 & 2.63 & 76 \\
\hline 5 & 2 & 3 & 178 & 3.5 & 0.062 & 29.40 & 1054.4 & 0.0561 & 16.95 & 4.994 & 0.074 & 3.06 & 76 & 2.96 & 76 \\
\hline 5 & 2 & 3 & 178 & 5.8 & 0.040 & 29.48 & 1066.5 & 0.0256 & 6.94 & 2.403 & 0.151 & 3.01 & 74 & 2.95 & 72 \\
\hline 5 & 2 & 3 & 59 & 1.65 & 0.040 & 9.02 & 1089.3 & 0.0405 & 7.79 & 4.521 & 0.076 & 2.65 & 78 & 2.64 & 77 \\
\hline 5 & 2 & 3 & 59 & 3.5 & 0.036 & 10.15 & 1093.6 & 0.0320 & 5.85 & 3.672 & 0.092 & 3.06 & 76 & 3.01 & 76 \\
\hline 5 & 2 & 3 & 59 & 5.8 & 0.021 & 9.76 & 1100.6 & 0.0128 & 2.30 & 1.481 & 0.228 & 3.01 & 74 & 2.99 & 73 \\
\hline 5 & 2 & 4 & 178 & 1.65 & 0.068 & 29.48 & 1704.3 & 0.0677 & 25.48 & 5.404 & 0.071 & 2.69 & 81 & 2.69 & 81 \\
\hline 5 & 2 & 4 & 178 & 3.5 & 0.056 & 29.48 & 1711.6 & 0.0489 & 16.99 & 4.058 & 0.093 & 2.91 & 76 & 2.93 & 76 \\
\hline 5 & 2 & 4 & 178 & 5.8 & 0.038 & 29.48 & 1722.5 & 0.0253 & 6.94 & 2.373 & 0.153 & 3.51 & 81 & 3.44 & 80 \\
\hline 5 & 2 & 4 & 59 & 1.65 & 0.040 & 9.10 & 1747.5 & 0.0405 & 7.86 & 4.501 & 0.076 & 2.69 & 81 & 2.69 & 81 \\
\hline 5 & 2 & 4 & 59 & 3.5 & 0.030 & 9.10 & 1751.6 & 0.0244 & 5.25 & 2.582 & 0.135 & 2.91 & 76 & 2.92 & 76 \\
\hline 5 & 2 & 4 & 59 & 5.8 & 0.017 & 9.10 & 1758.0 & 0.0105 & 2.14 & 1.132 & 0.305 & 3.51 & 81 & 3.48 & 80 \\
\hline 12 & 1 & 0 & 59 & 1.65 & 0.056 & 8.56 & 3.7 & 0.0559 & 7.40 & 4.863 & 0.066 & 2.87 & 16 & 3.05 & 19 \\
\hline 12 & 1 & 0 & 59 & 3.5 & 0.049 & 9.79 & 5.5 & 0.0448 & 5.64 & 3.999 & 0.080 & 2.02 & 20 & 2.11 & 23 \\
\hline 12 & 1 & 0 & 59 & 5.8 & 0.033 & 9.45 & 8.7 & 0.0219 & 2.22 & 2.174 & 0.141 & 1.89 & 11 & 1.88 & 15 \\
\hline 12 & 1 & 0 & 178 & 1.65 & 0.100 & 24.20 & 23.5 & 0.1000 & 20.92 & 6.914 & 0.050 & 2.87 & 16 & 3.47 & 26 \\
\hline 12 & 1 & 0 & 178 & 3.5 & 0.092 & 24.92 & 29.5 & 0.0851 & 14.37 & 6.545 & 0.051 & 2.02 & 20 & 2.27 & 29 \\
\hline 12 & 1 & 0 & 178 & 5.8 & 0.054 & 23.60 & 36.5 & 0.0337 & 5.55 & 2.625 & 0.127 & 1.89 & 11 & 1.87 & 21 \\
\hline 12 & 1 & 1 & 178 & 1.65 & 0.129 & 24.16 & 105.0 & 0.1294 & 20.88 & 10.187 & 0.033 & 5.18 & 53 & 4.36 & 40 \\
\hline 12 & 1 & 1 & 178 & 3.5 & 0.082 & 23.27 & 110.9 & 0.0617 & 13.41 & 4.181 & 0.084 & 2.91 & 53 & 2.62 & 42 \\
\hline 12 & 1 & 1 & 178 & 5.8 & 0.054 & 23.20 & 118.5 & 0.0355 & 5.46 & 2.857 & 0.115 & 1.81 & 43 & 1.84 & 34 \\
\hline 12 & 1 & 1 & 59 & 1.65 & 0.082 & 9.76 & 134.5 & 0.0820 & 8.43 & 8.081 & 0.038 & 5.18 & 53 & 4.74 & 46 \\
\hline 12 & 1 & 1 & 59 & 3.5 & 0.046 & 10.09 & 137.3 & 0.0333 & 5.82 & 2.523 & 0.133 & 2.91 & 53 & 2.77 & 48 \\
\hline 12 & 1 & 1 & 59 & 5.8 & 0.031 & 10.15 & 142.7 & 0.0204 & 2.39 & 1.892 & 0.166 & 1.81 & 43 & 1.82 & 40 \\
\hline 12 & 1 & 2 & 178 & 1.65 & 0.081 & 26.45 & 439.3 & 0.0815 & 22.86 & 4.865 & 0.075 & 5.16 & 71 & 5.16 & 67 \\
\hline
\end{tabular}




\begin{tabular}{|c|c|c|c|c|c|c|c|c|c|c|c|c|c|c|c|}
\hline 12 & 1 & 2 & 178 & 3.5 & 0.072 & 27.15 & 444.8 & 0.0649 & 15.65 & 4.182 & 0.085 & 4.24 & 56 & 3.93 & 55 \\
\hline 12 & 1 & 2 & 178 & 5.8 & 0.050 & 27.26 & 456.0 & 0.0341 & 6.42 & 2.483 & 0.137 & 2.50 & 49 & 2.36 & 48 \\
\hline 12 & 1 & 2 & 59 & 1.65 & 0.044 & 9.23 & 474.0 & 0.0442 & 7.98 & 3.284 & 0.103 & 5.16 & 71 & 5.16 & 69 \\
\hline 12 & 1 & 2 & 59 & 3.5 & 0.036 & 9.18 & 477.0 & 0.0312 & 5.29 & 2.398 & 0.140 & 4.24 & 56 & 4.09 & 56 \\
\hline 12 & 1 & 2 & 59 & 5.8 & 0.026 & 9.13 & 481.6 & 0.0177 & 2.15 & 1.604 & 0.198 & 2.50 & 49 & 2.44 & 49 \\
\hline 12 & 1 & 3 & 178 & 1.65 & 0.077 & 29.64 & 1072.6 & 0.0772 & 25.62 & 4.236 & 0.088 & 5.01 & 69 & 5.03 & 69 \\
\hline 12 & 1 & 3 & 178 & 3.5 & 0.069 & 29.64 & 1079.9 & 0.0625 & 17.09 & 3.776 & 0.096 & 5.74 & 65 & 5.51 & 64 \\
\hline 12 & 1 & 3 & 178 & 5.8 & 0.045 & 29.64 & 1090.9 & 0.0299 & 6.97 & 1.960 & 0.180 & 4.11 & 57 & 3.89 & 56 \\
\hline 12 & 1 & 3 & 59 & 1.65 & 0.040 & 10.15 & 1112.3 & 0.0405 & 8.77 & 2.751 & 0.127 & 5.01 & 69 & 5.02 & 69 \\
\hline 12 & 1 & 3 & 59 & 3.5 & 0.034 & 10.15 & 1116.0 & 0.0305 & 5.85 & 2.197 & 0.156 & 5.74 & 65 & 5.61 & 64 \\
\hline 12 & 1 & 3 & 59 & 5.8 & 0.024 & 10.15 & 1121.4 & 0.0158 & 2.39 & 1.291 & 0.254 & 4.11 & 57 & 4.01 & 57 \\
\hline 12 & 1 & 4 & 178 & 1.65 & 0.071 & 28.60 & 1682.1 & 0.0710 & 24.72 & 3.802 & 0.099 & 4.25 & 80 & 4.37 & 78 \\
\hline 12 & 1 & 4 & 178 & 3.5 & 0.066 & 28.52 & 1689.2 & 0.0617 & 16.44 & 3.776 & 0.096 & 5.18 & 89 & 5.26 & 85 \\
\hline 12 & 1 & 4 & 178 & 5.8 & 0.044 & 28.52 & 1700.9 & 0.0292 & 6.71 & 1.927 & 0.183 & 3.74 & 84 & 3.79 & 80 \\
\hline 12 & 1 & 4 & 59 & 1.65 & 0.039 & 9.37 & 1726.0 & 0.0387 & 8.10 & 2.677 & 0.130 & 4.25 & 80 & 4.31 & 79 \\
\hline 12 & 1 & 4 & 59 & 3.5 & 0.034 & 9.37 & 1729.5 & 0.0306 & 5.40 & 2.307 & 0.146 & 5.18 & 89 & 5.22 & 87 \\
\hline 12 & 1 & 4 & 59 & 5.8 & 0.023 & 9.37 & 1735.3 & 0.0158 & 2.20 & 1.333 & 0.243 & 3.74 & 84 & 3.76 & 82 \\
\hline 12 & 2 & 0 & 59 & 1.65 & 0.042 & 8.72 & 7.1 & 0.0424 & 7.54 & 3.186 & 0.106 & 2.18 & 36 & 2.31 & 40 \\
\hline 12 & 2 & 0 & 59 & 3.5 & 0.040 & 9.37 & 10.1 & 0.0388 & 5.40 & 3.293 & 0.098 & 2.73 & 20 & 2.96 & 27 \\
\hline 12 & 2 & 0 & 59 & 5.8 & 0.032 & 9.32 & 15.0 & 0.0244 & 2.19 & 2.580 & 0.117 & 2.53 & 26 & 2.78 & 33 \\
\hline 12 & 2 & 0 & 178 & 1.65 & 0.079 & 23.64 & 30.5 & 0.0786 & 20.43 & 4.872 & 0.074 & 2.18 & 36 & 2.53 & 46 \\
\hline 12 & 2 & 0 & 178 & 3.5 & 0.083 & 23.64 & 36.4 & 0.0826 & 13.63 & 6.431 & 0.052 & 2.73 & 20 & 3.26 & 36 \\
\hline 12 & 2 & 0 & 178 & 5.8 & 0.052 & 23.64 & 44.2 & 0.0337 & 5.56 & 2.622 & 0.127 & 2.53 & 26 & 2.98 & 39 \\
\hline 12 & 2 & 1 & 178 & 1.65 & 0.076 & 23.83 & 110.7 & 0.0759 & 20.59 & 4.604 & 0.079 & 3.38 & 69 & 2.92 & 56 \\
\hline 12 & 2 & 1 & 178 & 3.5 & 0.073 & 23.86 & 119.5 & 0.0715 & 13.76 & 5.153 & 0.066 & 4.40 & 69 & 3.83 & 52 \\
\hline 12 & 2 & 1 & 178 & 5.8 & 0.047 & 23.83 & 129.3 & 0.0308 & 5.61 & 2.285 & 0.148 & 3.83 & 62 & 3.44 & 51 \\
\hline 12 & 2 & 1 & 59 & 1.65 & 0.051 & 10.86 & 148.8 & 0.0514 & 9.39 & 3.798 & 0.089 & 3.38 & 69 & 3.13 & 62 \\
\hline 12 & 2 & 1 & 59 & 3.5 & 0.038 & 11.00 & 153.9 & 0.0312 & 6.34 & 2.191 & 0.158 & 4.40 & 69 & 4.13 & 61 \\
\hline 12 & 2 & 1 & 59 & 5.8 & 0.027 & 10.55 & 159.9 & 0.0183 & 2.48 & 1.573 & 0.205 & 3.83 & 62 & 3.69 & 58 \\
\hline 12 & 2 & 2 & 178 & 1.65 & 0.070 & 25.34 & 422.2 & 0.0699 & 21.90 & 3.946 & 0.094 & 3.71 & 89 & 3.61 & 83 \\
\hline 12 & 2 & 2 & 178 & 3.5 & 0.063 & 24.16 & 428.3 & 0.0574 & 13.93 & 3.681 & 0.097 & 4.70 & 88 & 4.62 & 83 \\
\hline 12 & 2 & 2 & 178 & 5.8 & 0.045 & 25.72 & 438.6 & 0.0310 & 6.05 & 2.215 & 0.155 & 2.94 & 86 & 3.15 & 80 \\
\hline 12 & 2 & 2 & 59 & 1.65 & 0.041 & 10.89 & 463.5 & 0.0413 & 9.41 & 2.731 & 0.129 & 3.71 & 89 & 3.66 & 86 \\
\hline 12 & 2 & 2 & 59 & 3.5 & 0.034 & 10.89 & 467.5 & 0.0295 & 6.28 & 2.027 & 0.172 & 4.70 & 88 & 4.66 & 86 \\
\hline 12 & 2 & 2 & 59 & 5.8 & 0.025 & 10.89 & 474.6 & 0.0174 & 2.56 & 1.431 & 0.229 & 2.94 & 86 & 3.01 & 84 \\
\hline 12 & 2 & 3 & 178 & 1.65 & 0.079 & 25.30 & 982.0 & 0.0786 & 21.86 & 4.710 & 0.077 & 3.99 & 83 & 3.93 & 84 \\
\hline 12 & 2 & 3 & 178 & 3.5 & 0.056 & 24.77 & 990.2 & 0.0444 & 14.28 & 2.480 & 0.150 & 5.70 & 89 & 5.52 & 89 \\
\hline 12 & 2 & 3 & 178 & 5.8 & 0.043 & 24.77 & 1002.4 & 0.0321 & 5.83 & 2.383 & 0.142 & 4.00 & 90 & 3.83 & 89 \\
\hline 12 & 2 & 3 & 59 & 1.65 & 0.038 & 11.76 & 1026.4 & 0.0384 & 10.17 & 2.358 & 0.153 & 3.99 & 83 & 3.96 & 83 \\
\hline 12 & 2 & 3 & 59 & 3.5 & 0.034 & 11.79 & 1032.2 & 0.0312 & 6.80 & 2.116 & 0.165 & 5.70 & 89 & 5.60 & 89 \\
\hline
\end{tabular}




\begin{tabular}{|c|c|c|c|c|c|c|c|c|c|c|c|c|c|c|c|}
\hline 12 & 2 & 3 & 59 & 5.8 & 0.025 & 10.86 & 1041.0 & 0.0173 & 2.56 & 1.421 & 0.231 & 4.00 & 90 & 3.94 & 90 \\
\hline 12 & 2 & 4 & 178 & 1.65 & 0.066 & 29.04 & 1619.2 & 0.0657 & 25.10 & 3.358 & 0.114 & 5.11 & 90 & 4.86 & 88 \\
\hline 12 & 2 & 4 & 178 & 3.5 & 0.062 & 28.17 & 1628.6 & 0.0587 & 16.24 & 3.532 & 0.103 & 5.39 & 88 & 5.45 & 88 \\
\hline 12 & 2 & 4 & 178 & 5.8 & 0.042 & 29.52 & 1642.8 & 0.0278 & 6.95 & 1.754 & 0.204 & 4.42 & 92 & 4.34 & 91 \\
\hline 12 & 2 & 4 & 59 & 1.65 & 0.036 & 11.59 & 1672.1 & 0.0365 & 10.02 & 2.200 & 0.165 & 5.11 & 90 & 4.95 & 89 \\
\hline 12 & 2 & 4 & 59 & 3.5 & 0.031 & 10.49 & 1679.8 & 0.0280 & 6.05 & 1.908 & 0.183 & 5.39 & 88 & 5.43 & 88 \\
\hline 12 & 2 & 4 & 59 & 5.8 & 0.020 & 10.26 & 1692.6 & 0.0131 & 2.41 & 0.961 & 0.353 & 4.42 & 92 & 4.39 & 92 \\
\hline 20 & 1 & 0 & 59 & 1.65 & 0.059 & 10.98 & 5.4 & 0.0590 & 9.49 & 3.604 & 0.092 & 2.73 & 16 & 3.35 & 20 \\
\hline 20 & 1 & 0 & 59 & 3.5 & 0.054 & 9.81 & 7.1 & 0.0498 & 5.66 & 3.617 & 0.087 & 3.55 & 10 & 3.80 & 14 \\
\hline 20 & 1 & 0 & 59 & 5.8 & 0.041 & 9.65 & 11.1 & 0.0305 & 2.27 & 2.741 & 0.107 & 2.20 & 18 & 2.39 & 22 \\
\hline 20 & 1 & 0 & 178 & 1.65 & 0.129 & 29.40 & 26.3 & 0.1294 & 25.41 & 7.154 & 0.048 & 2.73 & 16 & 4.50 & 26 \\
\hline 20 & 1 & 0 & 178 & 3.5 & 0.095 & 26.68 & 30.8 & 0.0771 & 15.38 & 4.227 & 0.081 & 3.55 & 10 & 4.19 & 20 \\
\hline 20 & 1 & 0 & 178 & 5.8 & 0.066 & 27.97 & 38.6 & 0.0454 & 6.58 & 2.923 & 0.112 & 2.20 & 18 & 2.58 & 27 \\
\hline 20 & 1 & 1 & 178 & 1.65 & 0.119 & 23.98 & 81.7 & 0.1189 & 20.72 & 6.974 & 0.048 & 8.56 & 48 & 6.14 & 35 \\
\hline 20 & 1 & 1 & 178 & 3.5 & 0.086 & 23.98 & 87.6 & 0.0695 & 13.82 & 3.817 & 0.090 & 5.53 & 42 & 4.77 & 30 \\
\hline 20 & 1 & 1 & 178 & 5.8 & 0.057 & 23.57 & 95.4 & 0.0372 & 5.55 & 2.357 & 0.139 & 3.25 & 42 & 2.89 & 34 \\
\hline 20 & 1 & 1 & 59 & 1.65 & 0.071 & 8.56 & 109.7 & 0.0713 & 7.40 & 5.426 & 0.057 & 8.56 & 48 & 7.20 & 40 \\
\hline 20 & 1 & 1 & 59 & 3.5 & 0.043 & 8.85 & 112.5 & 0.0316 & 5.10 & 1.928 & 0.172 & 5.53 & 42 & 5.15 & 36 \\
\hline 20 & 1 & 1 & 59 & 5.8 & 0.027 & 8.24 & 117.5 & 0.0171 & 1.94 & 1.247 & 0.251 & 3.25 & 42 & 3.12 & 39 \\
\hline 20 & 1 & 2 & 178 & 1.65 & 0.100 & 28.29 & 350.3 & 0.1000 & 24.45 & 4.954 & 0.072 & 7.72 & 58 & 8.01 & 54 \\
\hline 20 & 1 & 2 & 178 & 3.5 & 0.074 & 28.29 & 357.3 & 0.0602 & 16.31 & 2.831 & 0.128 & 7.32 & 71 & 6.74 & 62 \\
\hline 20 & 1 & 2 & 178 & 5.8 & 0.051 & 28.25 & 371.2 & 0.0348 & 6.65 & 1.955 & 0.175 & 4.08 & 67 & 3.85 & 60 \\
\hline 20 & 1 & 2 & 59 & 1.65 & 0.055 & 9.40 & 396.3 & 0.0545 & 8.12 & 3.462 & 0.095 & 7.72 & 58 & 7.87 & 56 \\
\hline 20 & 1 & 2 & 59 & 3.5 & 0.037 & 9.67 & 401.0 & 0.0292 & 5.58 & 1.636 & 0.209 & 7.32 & 71 & 7.07 & 67 \\
\hline 20 & 1 & 2 & 59 & 5.8 & 0.024 & 9.87 & 408.2 & 0.0157 & 2.32 & 0.999 & 0.328 & 4.08 & 67 & 4.02 & 65 \\
\hline 20 & 1 & 3 & 178 & 1.65 & 0.069 & 25.53 & 983.8 & 0.0688 & 22.06 & 2.973 & 0.125 & 6.31 & 78 & 6.52 & 74 \\
\hline 20 & 1 & 3 & 178 & 3.5 & 0.061 & 24.39 & 990.9 & 0.0554 & 14.06 & 2.695 & 0.133 & 6.01 & 80 & 6.19 & 79 \\
\hline 20 & 1 & 3 & 178 & 5.8 & 0.043 & 27.11 & 1001.5 & 0.0299 & 6.38 & 1.587 & 0.219 & 5.93 & 75 & 5.70 & 74 \\
\hline 20 & 1 & 3 & 59 & 1.65 & 0.033 & 9.45 & 1024.1 & 0.0334 & 8.17 & 1.656 & 0.215 & 6.31 & 78 & 6.43 & 76 \\
\hline 20 & 1 & 3 & 59 & 3.5 & 0.028 & 9.79 & 1028.9 & 0.0249 & 5.64 & 1.282 & 0.274 & 6.01 & 80 & 6.10 & 79 \\
\hline 20 & 1 & 3 & 59 & 5.8 & 0.020 & 9.92 & 1034.9 & 0.0138 & 2.34 & 0.823 & 0.407 & 5.93 & 75 & 5.85 & 74 \\
\hline 20 & 2 & 0 & 59 & 1.65 & 0.053 & 8.80 & 6.4 & 0.0526 & 7.61 & 3.386 & 0.096 & 3.34 & 38 & 3.28 & 42 \\
\hline 20 & 2 & 0 & 59 & 3.5 & 0.047 & 10.12 & 10.7 & 0.0437 & 5.83 & 2.926 & 0.110 & 2.19 & 33 & 2.61 & 40 \\
\hline 20 & 2 & 0 & 59 & 5.8 & 0.031 & 10.18 & 15.7 & 0.0205 & 2.39 & 1.471 & 0.214 & 2.04 & 50 & 2.44 & 53 \\
\hline 20 & 2 & 0 & 178 & 1.65 & 0.098 & 26.26 & 25.6 & 0.0977 & 22.69 & 4.969 & 0.071 & 3.34 & 38 & 3.17 & 49 \\
\hline 20 & 2 & 0 & 178 & 3.5 & 0.081 & 26.56 & 31.0 & 0.0705 & 15.31 & 3.705 & 0.094 & 2.19 & 33 & 3.06 & 47 \\
\hline 20 & 2 & 0 & 178 & 5.8 & 0.054 & 24.24 & 46.7 & 0.0359 & 5.70 & 2.203 & 0.150 & 2.04 & 50 & 2.79 & 56 \\
\hline 20 & 2 & 1 & 178 & 1.65 & 0.082 & 23.34 & 98.5 & 0.0815 & 20.18 & 4.012 & 0.089 & 2.80 & 75 & 3.00 & 61 \\
\hline 20 & 2 & 1 & 178 & 3.5 & 0.064 & 23.34 & 104.3 & 0.0536 & 13.46 & 2.621 & 0.136 & 4.80 & 76 & 3.90 & 61 \\
\hline 20 & 2 & 1 & 178 & 5.8 & 0.044 & 23.34 & 112.9 & 0.0298 & 5.49 & 1.704 & 0.199 & 3.91 & 65 & 3.34 & 60 \\
\hline
\end{tabular}




\begin{tabular}{|c|c|c|c|c|c|c|c|c|c|c|c|c|c|c|c|}
\hline 20 & 2 & 1 & 59 & 1.65 & 0.047 & 9.23 & 129.2 & 0.0470 & 7.98 & 2.791 & 0.120 & 2.80 & 75 & 2.91 & 67 \\
\hline 20 & 2 & 1 & 59 & 3.5 & 0.035 & 9.23 & 133.0 & 0.0287 & 5.32 & 1.631 & 0.209 & 4.80 & 76 & 4.36 & 69 \\
\hline 20 & 2 & 1 & 59 & 5.8 & 0.025 & 9.23 & 137.9 & 0.0175 & 2.17 & 1.214 & 0.262 & 3.91 & 65 & 3.70 & 63 \\
\hline 20 & 2 & 2 & 178 & 1.65 & 0.076 & 24.84 & 374.9 & 0.0759 & 21.47 & 3.493 & 0.104 & 5.43 & 76 & 4.44 & 76 \\
\hline 20 & 2 & 2 & 178 & 3.5 & 0.063 & 24.77 & 383.1 & 0.0554 & 14.28 & 2.675 & 0.134 & 4.91 & 84 & 4.87 & 81 \\
\hline 20 & 2 & 2 & 178 & 5.8 & 0.044 & 24.77 & 394.3 & 0.0304 & 5.83 & 1.698 & 0.201 & 3.79 & 76 & 3.83 & 73 \\
\hline 20 & 2 & 2 & 59 & 1.65 & 0.038 & 10.01 & 415.0 & 0.0384 & 8.65 & 1.980 & 0.177 & 5.43 & 76 & 4.83 & 76 \\
\hline 20 & 2 & 2 & 59 & 3.5 & 0.032 & 10.01 & 420.7 & 0.0273 & 5.77 & 1.451 & 0.240 & 4.91 & 84 & 4.89 & 83 \\
\hline 20 & 2 & 2 & 59 & 5.8 & 0.022 & 10.01 & 430.6 & 0.0147 & 2.35 & 0.900 & 0.368 & 3.79 & 76 & 3.80 & 75 \\
\hline 20 & 2 & 3 & 178 & 1.65 & 0.056 & 28.44 & 1055.3 & 0.0564 & 24.58 & 2.093 & 0.187 & 6.50 & 90 & 6.30 & 88 \\
\hline 20 & 2 & 3 & 178 & 3.5 & 0.063 & 28.44 & 1078.8 & 0.0635 & 16.40 & 3.061 & 0.117 & 6.62 & 89 & 6.37 & 88 \\
\hline 20 & 2 & 3 & 178 & 5.8 & 0.044 & 28.52 & 1064.7 & 0.0302 & 6.71 & 1.570 & 0.223 & 5.75 & 85 & 5.42 & 84 \\
\hline 20 & 2 & 3 & 59 & 1.65 & 0.036 & 11.24 & 1105.3 & 0.0365 & 9.71 & 1.731 & 0.209 & 6.50 & 90 & 6.38 & 89 \\
\hline 20 & 2 & 3 & 59 & 3.5 & 0.032 & 10.52 & 1111.5 & 0.0292 & 6.06 & 1.569 & 0.221 & 6.62 & 89 & 6.48 & 89 \\
\hline 20 & 2 & 3 & 59 & 5.8 & 0.019 & 10.46 & 1119.7 & 0.0122 & 2.46 & 0.662 & 0.521 & 5.75 & 85 & 5.64 & 85 \\
\hline
\end{tabular}

The velocities measured are from the points indicated $(1.65,3.5, \& 5.8)$ to bottom of flume

Sectional Velocity and discharge is relative to within each of the three sections (upper, mid, and lower)

Interpolated random roughness and rock covers are based on time of measurement of velocities for each section vs. time actuall y measured. 